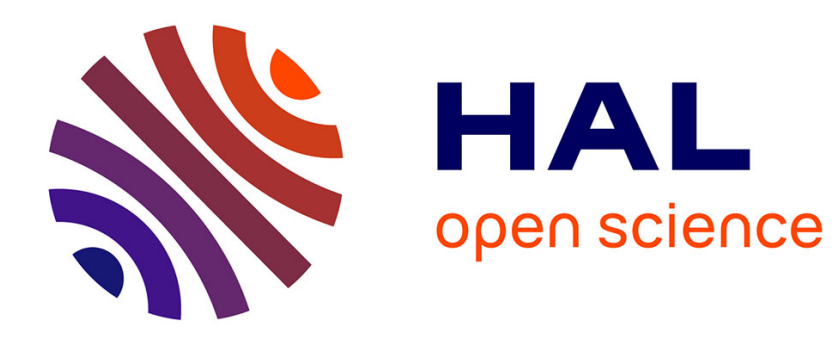

\title{
Biologie et filiation: les habits neufs de l'ordre naturel
}

Daniel Borrillo

\section{To cite this version:}

Daniel Borrillo. Biologie et filiation: les habits neufs de l'ordre naturel. Contemporary French Civilization, 2014, Au-delà du mariage, 39 (3), 10.3828/cfc.2014.18 . hal-01225160

\section{HAL Id: hal-01225160 https://hal.science/hal-01225160}

Submitted on 12 Nov 2015

HAL is a multi-disciplinary open access archive for the deposit and dissemination of scientific research documents, whether they are published or not. The documents may come from teaching and research institutions in France or abroad, or from public or private research centers.
L'archive ouverte pluridisciplinaire HAL, est destinée au dépôt et à la diffusion de documents scientifiques de niveau recherche, publiés ou non, émanant des établissements d'enseignement et de recherche français ou étrangers, des laboratoires publics ou privés. 


\section{Biologie et filiation: les habits neufs de l'ordre naturel}

Dès l'origine, le débat sur le droit au mariage pour les couples de même sexe s'est focalisé non pas tant sur la question du couple que sur celle de la filiation entendue comme l'ordre symbolique de la différence des sexes garant de la bonne structuration à la fois de l'individu et de la Civilisation. L'ouverture du mariage aux couples de même sexe a mis à mal cet ordre symbolique. C'est pourquoi, la différence des sexes doit être désormais instituée autrement. L'accès aux origines biologiques et la place grandissante de la preuve génétique dans les contentieux familiaux garantissent cette exigence conservatrice et permettent, à partir du socle biologique, de refonder en nature l'ordre de la parenté. A défaut d'avoir pu empêcher l'ouverture du mariage, les conservateurs tentent de préserver l'ordre symbolique de la différence des sexes avec la biologisation des liens de filiation.

From the start, the debate on the right to marriage for same-sex couples has focused less on the question of conjugality than on that of filiation, defined as the symbolic order of sexual difference that guarantees the correct structuring of both individual and Civilization. The opening of marriage to same-sex couples has undone this symbolic order. This is why sexual difference must now be instituted differently. Access to biological origins and the growing importance of genetic proof in family disputes are the answer to this conservative imposition, making it possible to provide a new foundation to the order of kinship on a biological basis. Conservatives may not have prevented the opening of marriage; but they still try to preserve the symbolic order of sexual difference through the biologisation of filiation.

\section{Introduction}

Tout au long du débat parlementaire sur le mariage pour tous et même après l'adoption définitive de la loi, ${ }^{1}$ la doxa familialiste n'a pas cessé de manifester sa vive opposition à cette réforme majeure du code civil. La contestation prend forme le 15 août 2012, jour de l'Assomption de la Vierge Marie, 
lorsque Monseigneur André Vingt-trois, président de la Conférence des évêques de France, fait parvenir une lettre aux paroisses incitant les fidèles à prier pour la sauvegarde de la famille traditionnelle. Depuis lors, plusieurs manifestations se sont organisées dans les principales villes de l'Hexagone et les veilleurs continuent à rôder devant le ministère de la Justice.

L'opposition s'est articulée autour de la défense d'une conception du mariage plus proche du droit canonique que du droit civil en associant l'union matrimoniale à sa supposée finalité reproductive. Ainsi, un certain nombre de slogans, brandis dans ces cortèges, soulignaient le caractère évident et naturel des liens de filiation. On pouvait effectivement lire dans les pancartes:

" Mariage $=1$ homme + une femme ». "Tous nés d'un homme et d'une femme "; " 1 père +1 mère, c'est élémentaire ". " 1 papa 1 maman, on ne ment pas aux enfants ", "Non à la filiation-fiction " ou encore : "Y a pas d'ovules dans les testicules ». ("Manif pour tous »)

Ces formules furent relayées par les médias (BFM TV 1) et répétées ad nauseam par l'opposition dans l'enceinte parlementaire. ${ }^{2}$ Malgré leur extrême simplicité (ou plutôt grâce à elle), ces slogans ont été assez efficaces ${ }^{3}$ aussi bien sur le plan politique que juridique. D'une part, ils ont permis de mieux masquer politiquement l'homophobie derrière l'argument de l'intérêt de l'enfant (entendu comme droit à avoir un père et une mère). D'autre part, avant même le début de la discussion parlementaire du projet de loi, la Commission des lois de l'Assemblée nationale, pour contrer l'opposition, a voté un amendement "balai " permettant de maintenir dans la loi les termes "père » et "mère ». L'expression "parent » est retenue uniquement pour les couples de personnes de même sexe.

Dans la version originelle, les mots "père " et "mère " étaient systématiquement remplacés par celui de "parents ». Cela constituait une véritable avancée symbolique car la loi n'a pas pour mission de signifier la nature sexuée du parent mais simplement sa fonction parentale. En effet, si les hommes et les femmes ont les mêmes droits et si les rôles parentaux sont aujourd'hui interchangeables, pourquoi faut-il maintenir la distinction terminologique dans le code civil? ${ }^{4}$

La question de la différence des sexes a toujours été intrinsèquement liée à la filiation. Ainsi, au fur et à mesure que les couples de même sexe commencent à jouir d'un certain nombre de droits parentaux, se développe un mécanisme politico-juridique caractérisé par une forme de retour au biologique en matière de filiation.

Pour le conservatisme de droite, il est question de combattre toute forme de reconnaissance des familles en dehors de celle constituée par le mariage 
hétérosexuel et la filiation légitime. Une résolution du Conseil municipal de Fontgombault du 24 octobre 2013 refusant le mariage des couples de même sexe va jusqu' à invoquer "une loi naturelle supérieure aux lois humaines ". Le raisonnement des élus de la commune se fonde sur l'idée que les couples homosexuels sont « radicalement incapables de procréer un être humain qui soit issu de cette union et par conséquent de l'éduquer à titre de parents dans l'altérité et la complémentarité " ("La justice »1). Sans aller jusqu'à invoquer l'ordre naturel, les députés et sénateurs de droite ont défendu systématiquement le modèle de la famille traditionnelle biparentale.

Pour le conservatisme de gauche, il est question d'intégrer les nouvelles formes familiales tout en maintenant certaines interdictions (assistance médicale à la procréation [AMP] pour les couples homosexuels et gestation pour autrui [GPA] pour l'ensemble des couples) ou tout au moins (si un jour ces techniques de reproduction venaient à être autorisées) en préparant les réformes législatives nécessaires pour inscrire lesdites techniques dans le droit sans bouleverser le primat de la différence des sexes. Tout se passe comme si, pour pouvoir reconnaitre juridiquement l'homoparenté, il fallait signifier, d'une manière ou d'une autre, la prééminence de l'hétéroparenté. L'enjeu symbolique relève bien de la parenté et non pas de la parentalité car il s'agit de l'institution juridique de la filiation elle-même et non pas seulement de l'autorité parentale ou du statut du beau-parent.

En ce sens, les pancartes de la "Manif pour tous $\|^{5}$ et la décision du Conseil municipal de Fontgombault ne constituent que la partie émergée de l'iceberg ainsi que ses manifestations les plus paroxystiques. La partie invisible risque de perpétuer, d'une manière bien plus insidieuse, la hiérarchie des parentés.

Pourtant, depuis le début du $\mathrm{XX}^{\text {ème }}$ siècle, l'ensemble des travaux sociologiques démontrent que la famille n'est ni naturelle ni intangible. Depuis les années 70, le droit n'a pas cessé de dissocier le mariage de la filiation, en particulier pour donner aux "bâtards " les mêmes droits qu'aux enfants légitimes.

De surcroit, tous les indicateurs démographiques manifestent le caractère polymorphe des familles. Pourtant, l'adhésion à un discours simpliste sur la famille nucléaire, présentée comme le modèle unique à suivre, n'a jamais occupé une place aussi importante dans l'espace public français.

Comment ce discours d'évidence, qui fonde l'imaginaire de la filiation sur la biologie, est-il réactualisée? D'où vient cette forme d'essentialisme qui s'acharne à ne pas effacer de la loi la différence de sexes?

Dans les pages qui suivent, nous allons essayer d'analyser les composantes de ce montage idéologique en apparence complexe. Cette analyse est d'autant 
plus nécessaire que les enjeux vont bien au-delà de l'égalité des familles homoparentales: il s'agit des fondements politiques du droit civil de la filiation.

\section{Reproduction et filiation}

Alors que la reproduction est un fait biologique, la filiation apparait comme une institution éminemment juridique (Borrillo, "Le droit en matière de reproduction »). Le droit peut certes tenir compte du fait naturel, mais seulement en tant que dispositif d'agencement parental: la filiation répond à des règles propres, affranchies de la nature. Comme le soulignait le doyen Cornu: "Le droit de la filiation n'est pas seulement un droit de la vérité (biologique), c'est aussi un droit de la vie, de l'intérêt de l'enfant, de la paix des familles, des affections, des sentiments moraux, de l'ordre établi, du temps qui passe..." (270).

Le droit moderne rompt avec le naturalisme du droit canonique. ${ }^{6}$ Désormais, sur le plan juridique, ce ne sont pas tant les racines naturelles des institutions qui comptent mais la réalisation d'objectifs spécifiques (telos) comme la paix des familles, la transmission du patrimoine, la solidarité des générations ou la protection des plus faibles. Le droit moderne s'inscrit ainsi dans la tradition du droit romain pour lequel la filiation permettait surtout d'organiser les successions et les tutelles ou l'interdiction des témoignages contre les parents dans la juridiction criminelle et non pas d'octroyer à l'enfant une quelconque identité psychologique. ${ }^{7}$

Pour le modèle civiliste, nul besoin donc de la biologie ni de la différence des sexes pour inscrire l'enfant dans une lignée (Borrillo, "La parenté et la parentalité dans le droit »). L'adoption plénière ouverte aux célibataires ou la possession d'état ("Etablissement de la filiation » 1) constituent les exemples les plus paradigmatiques de cette dissociation. Dans ces deux cas, la filiation peut être établie à l'égard d'une ou deux personnes qui n'ont aucun lien biologique avec l'enfant.

La différence des sexes comme condition sine qua non de l'établissement du droit de la filiation n'apparaît dans le débat politique de manière explicite que très récemment, plus précisément dans un rapport commandé à la fin des années 1980 par Michel Rocard et au sein duquel il est prôné « l'affirmation de la valeur des structures naturelles de la parenté "(Conseil d'État). Cherchant à trouver un encadrement pour les techniques procréatives émergentes, un principe étonnant sera alors énoncé dans ledit rapport : «deux parents, pas un de plus, pas un de moins " (Conseil d'État 13). 
La "panique morale " exprimée dans ce document s'explique non seulement par l'avènement spectaculaire des techniques procréatives mais aussi et surtout par l'éventualité d'une parenté exclusivement féminine ou exclusivement masculine que lesdites techniques pourraient entraîner. Pourtant, cette assignation unisexuée de la filiation existe déjà depuis la loi $n^{\circ}$ 66-500 du 11 juillet 1966 portant réforme de l'adoption. En effet, ce texte consacre la famille monoparentale adoptive par la possibilité d'une adoption plénière individuelle (c'est-à-dire la rupture avec la famille par le sang et la création d'un lien avec l'adoptant). Mais, à l'époque, la "menace " de l'homoparentalité ne planait pas. Celle-ci " risque " effectivement de transformer l'assignation unisexuée de la loi de 1966, en une assignation homosexuée. C'est en fait l'homosexualité, et non pas la monoparentalité, qui effraye tant la pensée conservatrice.

\section{L'architecture clinique et hétérocentrée de l'Assistance Médicale à la Procréation (AMP)}

Anticipant la revendication de futurs parents gays et lesbiens et surtout pour mieux la contrer, les lois bioéthiques ont construit l'AMP comme un supposé " remède " contre l'infécondité sur la base de la simulation de l'acte hétérosexuel procréatif. Celui-ci apparaît désormais comme le point de départ permettant la mise en place d'un schéma symbolique: la vraisemblance biologique.

L'AMP n'a pas été pensée politiquement ni construite juridiquement à partir d'un droit subjectif ou d'une liberté (comme ce fut le cas pour la contraception ou l'IVG) mais comme un acte médical qui sert à pallier une stérilité ou à éviter la transmission d'une maladie grave. Ainsi, l'AMP constitue en quelque sorte une démission du politique en faveur de l'expertise clinique, laquelle déterminera la frontière du permis et de l'interdit en la matière.

A cette raison médicale s'ajoute la promotion d'un type d'agencement familial: le couple hétérosexuel en âge de procréer. En France, la législation sur l'AMP est légitimée par cette conception d'une parenté "naturelle ", fondée sur l'idée que l'intérêt du futur enfant implique le droit d'avoir une mère et un père et des liens biologiques vrais ou vraisemblables avec les personnes remplissant cette tâche sociale. Ces valeurs, décrites comme universelles, se trouvent, de surcroît, à l'origine de l'inclusion de l'AMP parmi les services remboursés par la Sécurité sociale.

L'article L2141-2 du Code de la santé publique statue en effet : 
L'assistance médicale à la procréation a pour objet de remédier à l'infertilité d'un couple ou d'éviter la transmission à l'enfant ou à un membre du couple d'une maladie d'une particulière gravité. Le caractère pathologique de l'infertilité doit être médicalement diagnostiqué. L'homme et la femme formant le couple doivent être vivants, en âge de procréer et consentir préalablement au transfert des embryons ou à l'insémination. Font obstacle à l'insémination ou au transfert des embryons le décès d'un des membres du couple, le dépôt d'une requête en divorce ou en séparation de corps ou la cessation de la communauté de vie, ainsi que la révocation par écrit du consentement par l'homme ou la femme auprès du médecin chargé de mettre en œuvre l'assistance médicale à la procréation. (Code de la santé publique)

La peur d'une filiation homosexuée a eu comme résultat la mise en question radicale de la notion juridique de filiation, laquelle sera conçue désormais davantage comme un événement naturalisable que comme l'expression de la volonté individuelle ou de celle du couple.

La crainte de l'institutionnalisation de l'homoparenté et la foi dans la preuve biologique (sanguine ou génétique) affaiblissent les autres figures de la filiation reposant sur la volonté et sur le vécu. En effet, outre les lois bioéthiques, la place prépondérante de la vérité biologique dans l'établissement du lien filial fut confirmée par les magistrats dans un célèbre arrêt de la Cour de cassation du 28 mars 2000. Ainsi, la distinction traditionnelle entre reproduction (fait biologique) et filiation (fait juridique), ${ }^{8}$ fondement du droit civil moderne, se trouve profondément questionnée. Non seulement à partir d'arguments classiques provenant du droit canon (ordre naturel, Loi supérieure) mais aussi et surtout par l'émergence d'une rhétorique nouvelle qui, d'une part, fera de la différence des sexes l'arrière-plan de l'ensemble des filiations et, d'autre part, placera l'expertise sanguine et la preuve d'ADN au cour du dispositif judiciaire de la parenté.

Les grands principes autour desquels s'articule encore le droit civil, tels l'autonomie de la volonté, le respect de la vie privée, l'égalité ou la libre disposition de soi, se trouvent ainsi fragilisés au profit des nouvelles prescriptions (étrangères au droit civil) fondées sur des notions telles que la bonne structuration psychique de l'enfant (voire de l'humain) ${ }^{9}$ ou la valeur éminente de la différence des sexes (Agacinski-Jospin 1)

Éric Fassin a bien analysé l'évolution du discours théologique en la matière, en montrant comment le pape Benoît XVI a superposé l'ordre naturel avec les lois de la nature pour refonder l'ordre sexuel en nature (Fassin, "Les "forêts tropicales" »). De même, le conservatisme de gauche va superposer l'ordre symbolique de la différence des sexes à la vérité des origines pour refonder 
l'ordre parental sur la prééminence de l'hétérosexualité. Tout se passe comme si derrière l'homoparenté ou la monoparenté, le droit devait nécessairement reconnaitre l'hétéroparenté.

\section{Le nouveau montage idéologique}

La dimension conventionnelle de la filiation, fiction instituée par le droit, ne posait guère de problèmes lorsque celle-ci pouvait fonctionner comme un artifice imitant la nature. La vraisemblance biologique du couple d'adoptants avec le couple des géniteurs permettait d'assumer la création d'un lien de filiation complètement dissocié de la vérité biologique. On pouvait instituer le vivant - Vitam Instituere pour reprendre l'expression du droit romain vulgarisée par Pierre Legendre - dès lors que la base de ladite institutionnalisation était effectivement l'hétérosexualité.

Aujourd'hui, grâce à la loi permettant l'adoption pour les couples de même sexe, le droit ne peut plus faire comme si les parents d'intention coïncident avec le couple procréatif comme c'était le cas lorsque seulement les couples hétérosexuels pouvaient adopter. Mais, plutôt que d'assumer la dimension conventionnelle de la filiation et dire que c'est la volonté qui fonde l'ordre parental, le néo-biologisme s'acharnera à le refonder sur la base de la vérité des origines.

Comme l'homoparenté ne peut reposer sur un montage dont le point de départ est la ressemblance biologique, au lieu de s'affranchir de celle-ci en affirmant la dimension conventionnelle de toute filiation, il est question désormais de creuser encore plus profondément dans ce paradigme naturel de la ressemblance biologique pour trouver le point de départ qui faciliterait la mise en place d'un schéma symbolique inédit, certes, mais toujours plus proche de la nature. Pour ce faire, il faut, d'une part, dénoncer la ressemblance biologique considérée comme de l'ordre du pseudo-procréatif et, d'autre part, octroyer aux origines une place juridique. Ainsi, un personnage nouveau fait son apparition sur la scène juridique: le géniteur. La pensée néo-biologiste prend ainsi le relais des slogans de la "Manif pour tous ", en considérant indispensable d'organiser l'ordre de la filiation sur un ancrage "solide". Désormais, il faudra enraciner la filiation dans le tréfonds sécurisant de la "vraie » biologie. A la différence des manifestants contre le mariage pour tous, les experts se gardent bien d'assimiler la filiation à la procréation. Leur geste n'est pas pour autant moins biologisant. En effet, à l'argument de la vraisemblance biologique utilisé pour justifier l'adoption et l'AMP 
hétérosexuelles, ces experts opposeront la certitude des origines génétiques. À l'heure où la filiation risque d'être «brouillée " par la filiation monosexuée, il est question d'assurer la permanence de l'Humain à travers le marquage de ses origines. Paradoxalement (ou tout au moins en apparence), c'est justement en s'appuyant sur l'homoparenté que le nouveau montage idéologique prendra forme. Celle-ci n'apparait plus comme impensable ou terrifiante mais, au contraire, comme l'événement permettant de repenser la filiation adoptive ou par AMP non plus sur la base de la ressemblance naturelle qui n'est que mensonge mais sur celle de la certitude biologique de l'engendrement. Ainsi, le droit n'aurait plus besoin de la vraisemblance biologique pour construire le nouvel ordre de la parenté. La vérité de l'engendrement lui permet d'ancrer la filiation sans ambiguiité dans une assise biologique, à une seule et unique condition: la levée de l'anonymat des géniteurs (Conseil d'État 2013). ${ }^{10}$

Loin d'être bannie (comme à l'époque du Pacs), l'homoparenté jouera désormais un rôle capital dans le nouveau dispositif. Elle devient ainsi la "fiction raisonnable " contre les mensonges de l'AMP et l'adoption plénière hétérosexuelles. L'homoparenté constitue ainsi une "fiction crédible " puisqu'incapable de gommer les origines, présentées désormais comme l'" identité narrative ", expression empruntée abusivement à Paul Ricœur pour mieux dissimuler les aspects biologisants de cette entreprise.

L'homoparenté, si le statut des géniteurs venait à être dévoilé comme le propose le rapport Théry (Théry et Leroyer), permettrait ainsi d'abolir la fiction "pseudo-procréative " d'une certaine hétéroparenté (adoption, AMP avec donneur, accouchement sous X...) en restaurant une fiction fondée non pas sur la vraisemblance biologique mais sur la vérité de l'engendrement (Théry et Leroyer). Et ceci afin de relier l'histoire de l'humain à l'origine, à la fois biologique et symbolique, de l'Humanité (Legendre, "Leçon IV »). Une fois le statut du géniteur reconnu par la loi civile, l'homoparenté n'apparaîtrait plus comme opposée à la loi naturelle mais, en revanche, comme la confirmant.

La Commission nationale consultative des Droits de l'Homme (CNCDH 2013) particulièrement séduite par ce dispositif, souligne que:

La fiction que l'adoption plénière institue est qualifiée de "mensonge institutionnalisé," auquel on opposerait le droit de l'enfant à connaître ses origines. Un tel “mensonge" n'est pourtant pas propre aux couples de personnes de même sexe qui, bien au contraire, sont dans une situation telle qu'il n'a pas lieu d'être. La critique vaut ainsi essentiellement pour l'adoption par des couples de sexes opposés et met en débat la question de l'adoption plénière elle-même qui coupe les liens avec la filiation d'origine et permet une 
substitution totale dans les actes d'état civil. (par. 22)

Le projet de loi invite dès lors à s'interroger sur l'accès aux origines sans que cela ne soit, encore une fois, propre à l'adoption par des couples de même sexe. (...) la CNCDH recommande que la réflexion sur l'accès aux origines soit prise en compte dans une réforme du droit de la famille. (par. 23)

$\mathrm{La} \mathrm{CNCDH}$ entend les arguments consistant à dire que la différence des sexes revêt un caractère fondamental dans la construction de sa filiation par un individu et que toute la prudence s'impose pour ne pas trop entamer la représentation psychique des deux lignées d'engendrement. A cet égard, la CNCDH recommande que l'établissement de la filiation dans un couple homoparental n'institue pas un enfant comme "né » de ce couple, que la réalité biologique de l'engendrement ne soit pas occultée. La CNCDH recommande pour toutes ces raisons qu'une réforme de l'adoption plénière soit engagée. (par. 25)

L'avis de la CNCDH suit l'opinion de plusieurs psychanalystes, anthropologues, et sociologues qui, lors des auditions parlementaires, avaient demandé l'inscription des géniteurs dans la loi. C'est pourquoi Monseigneur Vingt-Trois, le Grand Rabbin de France, le Président du culte musulman, le représentant des Evêques orthodoxes, ou la Fédération protestante n'ont pas eu besoin de mobiliser d'arguments théologiques: ceux utilisés par les gardiens de l'ordre symbolique "laï " suffisent car leur soubassement idéologique est commun. La réalité biologique de la différence des sexes doit être inscrite dans la Loi puisqu'elle donne sens, non seulement à la filiation, mais à la relation humaine tout entière.

Lorsque la CNCDH demande que la vérité biologique de l'engendrement ne soit pas occultée, elle participe à cette refondation de l'ordre de la parenté.

Le géniteur sera désormais appelé « donneur d'engendrement » en devenant un personnage central du dispositif néo-biologique. Ainsi, l'accès aux origines ne se résume nullement à la simple connaissance des personnes qui ont participé à la conception de l'enfant (connaissance qui nous semble parfaitement légitime). La question des origines va bien au-delà du droit à connaître ses géniteurs pour devenir la clé de voûte du rapport entre l'institutionnel et le corps (si cher à Pierre Legendre) : "Cela n'engage rien moins que la condition de mortalité situant chacun de nous à l'intérieur d'un monde humain signifiant qui a commencé avant notre naissance, qui continuera après notre mort, et dans lequel nous devons passer notre vie " (Théry 6) Ce n'est pas Monseigneur Vingt-Trois qui s'exprime de la sorte mais la sociologue qui préside l'un des groupes de travail mis en place par la ministre de la famille afin de préparer la réforme du droit de la filiation. Le 
groupe de travail en question - à l'origine du rapport Théry - prend le titre évocateur de "Filiation, origines, parentalité » dont la finalité serait de garantir l'accès aux origines biologique des personnes. L'association de la question des origines biologiques à la filiation et à la parentalité n'est nullement innocente. Rapprocher la question des origines d'une réforme du droit de la parenté dévoile cette entreprise consistant à établir une sorte de "filiation de souche " qui renvoie à la reproduction, tandis les autres formes de filiation viendraient s'ajouter, une fois établie dans la loi ce "nécessaire " arrière-plan biologique grâce à la levée de l'anonymat.

Dans ce contexte, la question des origines ne fait que réactiver les vieux mécanismes du jusnaturalisme consistant à fonder les institutions juridiques non pas sur la délibération démocratique mais sur certains fondements, prétendument invariables, et auxquels la volonté doit impérativement se soumettre (la Loi naturelle) sous peine de compromettre la coexistence démocratique par un relativisme individualiste.

\section{Les habits neufs de l'ordre naturel}

Les habits neufs de l'ordre naturel, entendu à la fois comme discours fondé sur un principe immuable (le biologique) et permettant la perpétuation d'une Métaphysique de la différence de sexes, trouve son origine dans le montage idéologique que nous venons d'analyser mais aussi à travers une pratique juridique caractérisée par la prééminence de la preuve sanguine et génétique dans le contentieux de la filiation. Depuis le 28 mars 2000, suite à une décision de la Cour de cassation, l'expertise sanguine est devenue un droit en matière de filiation (Borrillo, "La vérité biologique contre l'homoparentalité "). Avant cet arrêt, ladite expertise n'était pas systématique: elle relevait de l'appréciation souveraine des juges du fond. Ce renversement jurisprudentiel apparaît clairement exprimé dans les propos de l'avocate générale Cécile Petit: "L'incertitude identitaire quant à la filiation est pour un enfant source de graves déséquilibres sur le plan de la construction de sa personnalité " (Petit 5). L'engendrement devient ainsi la certitude identitaire qui devrait fonder la filiation.

Un arrêt du 12 mai 2004 (Cour de Cassation, pourvoi n 02-16152) a franchi un pas important en matière d'action en recherche de paternité en n'exigeant plus des présomptions ou indices graves pour rapporter la preuve de la paternité et en permettant la preuve directe par l'expertise.

La revalorisation de l'adoption simple au détriment de l'adoption plénière 
participe également de cette mouvance. Outre le rapport de la $\mathrm{CNCDH}$ inspiré par le conservatisme de gauche (Fine; Delaisi de Parseval), ${ }^{11}$ la doctrine de juristes commence à revendiquer l'adoption simple pour les couples de même sexe. La reconnaissance en France de l'adoption plénière effectuée à l'étranger par un couple homosexuel apparait pour la Cour de cassation comme contraire à l'ordre public international, au motif qu'elle aboutirait à la délivrance d'actes d'état civil masquant l'origine hétérosexuée de l'enfant (Cour de Cassation 2012). Une partie de la doctrine des juristes (inspiré par le néo-biologisme) a vu dans cette décision une avancée, car si l'origine biologique n'était pas masquée, il n'y aurait pas d'obstacle à la reconnaissance de l'adoption homoparentale. En effet, comme le note Louis d'Avout:

Dans ces affaires internationales qui, porosité des frontières oblige, ne diffèrent guère de leurs homologues internes françaises, la Cour de cassation esquisse rationnellement ce que pourrait être la réforme équilibrée voulue par le président: un rehaussement mesuré du droit des couples de même sexe à élever, comme les siens, les enfants qui par le sang sont étrangers à l'un ou aux deux des prétendants parents. Ceci passerait par la reconnaissance d'un droit à l'adoption simple, qui ajoute à la filiation naturelle, non pas à l'adoption plénière, qui efface la filiation naturelle non vécue et lui substitue un équivalent juridique assumé. (1973)

De même, le rapport Théry propose, en matière d'adoption plénière « de ne plus remplacer un acte de naissance par un autre, mais de garder un seul acte, l'acte de naissance originel. L'enfant ne naît pas une deuxième fois, l'adoption n'est pas un effacement de la naissance et de la vie antérieure. L'adoption devrait être transcrite sur l'acte de naissance d'origine, par une mention des noms actuels du ou des parents » (70).

La CNCDH, le rapport Théry, et la doctrine des juristes trouvera ainsi en Frigide Barjot une alliée de taille car selon la passionaria anti-mariage pour tous : "seule une loi ouvrant aux couples homos mariés le droit à une adoption simple - et non pas plénière car cela reviendrait à effacer la filiation biologique et écraser la nature - serait susceptible d'apaiser mes troupes " (Ecoiffier).

La personnification du don de gamètes et le statut du géniteur constituent le troisième pilier du néo-biologisme. Dans ce contexte, le modèle de l'AMP hétérosexuelle (appelé par les conservateurs de gauche "pseudo-procréatif ") est dénoncé comme étant un déni des dons et des donneurs. Le donneur devrait désormais être considéré comme une "personne singulière " puisqu'il ne donne plus une substance de son corps mais la vie elle-même. 
Or, en réalité, il n'est pas exact que ces gamètes soient nécessairement destinés à l'engendrement. Ils peuvent être affectés à la recherche scientifique ou tout simplement être détruits. En effet, la loi autorise la recherche sur l'embryon, sur les cellules souches embryonnaires et fotales humaines ainsi que sur les embryons conçus in vitro dans le cadre d'une assistance médicale à la procréation et qui ne font plus l'objet d'un projet parental.

Considérer ceci comme de l'engendrement est contraire non seulement au droit positif mais à l'évolution même du statut de l'embryon en France.

Cette personnification des produits du corps nous renvoie aux vieilles controverses philosophiques sur le statut de la semence et sa capacité à porter en elle la vie de l'embryon. Souvenons-nous de la doctrine aristotélicienne selon laquelle le sperme du mâle contient le principe de la forme tandis que celui de la femelle n'a que la matière. Si l'individu cesse d'être donneur d'un simple produit du corps (sperme ou ovocyte) pour devenir donneur d'engendrement, cela voudrait dire que la loi doit faire comme si les gamètes contenaient en eux-mêmes la vie humaine. Cette personnification des donneurs nous amène aux tréfonds de l'imaginaire religieux. En effet, comment ne pas penser au récit biblique sur la mort d'Onan pour «avoir laissé la semence se perdre à terre " (Livre de la Genèse. 38, 8-10), récit qui est à l'origine de la condamnation de la masturbation masculine. Même les autorités théologiques n'osent plus revendiquer cette conception de la reproduction. En effet, elles placent l'origine de la vie non pas dans les gamètes eux-mêmes mais dans la fécondation (Paul VI). Comme souvent, la réactualisation d'une pensée devient plus conservatrice que l'original.

Enfin, la levée de l'anonymat des donneurs apparait dans le contexte politique actuel non pas tant comme un droit de l'individu à connaitre ses origines mais comme un droit de l'Humanité « à la non falsification de la filiation ". Le rapport Théry va même jusqu'à proposer d'inscrire les origines biologiques dans l'acte de naissance "visant à le considérer comme le conservatoire de l'identité civile des personnes, autrement dit le garant que cette identité ne sera pas manipulée " (Théry et Leroyer 60). Il ne s'agit pas tant d'un droit individuel mais d'un impératif anthropologique: "C'est pourquoi ce que l'on nomme le droit d'accès aux origines est d'abord le droit pour l'enfant de ne pas être mis à part de l'humanité commune en étant transformé symboliquement en origine de soi-même. Cette transformation de l'individu en "origine de soi-même" est une atteinte profonde aux droits de la personne" (Théry et Leroyer 220).

La primauté de la preuve génétique dans le contentieux de la filiation, la prééminence de l'adoption simple, la levée de l'anonymat et le statut des 
géniteurs constituent les habits neufs de l'ordre naturel: voilà qui permettrait de donner à la filiation un ancrage solide (biologique) à partir duquel la fiction juridique puisse opérer tout en respectant l'ordre symbolique hétérosexuel.

Le projet de loi sur la famille, en cours d'élaboration au sein du Ministère des affaires sociales et sur la base du rapport Théry, reprend à son compte les éléments que nous venons de décrire. "Aujourd'hui, ce n'est plus le couple mais l'enfant qui fait la famille " (Bertinotti): la ministre déléguée de la famille annonce sans ambiguïté la philosophie de la réforme en excluant les couples de même sexe de l'accès à l'AMP et en associant explicitement la question des origines à la réforme de la filiation. Face aux pressions des groupes conservateurs le gouvernement a réduit le texte à certains aspects minimalistes comme les aménagements de certaines fonctions des beaux-parents sans pour autant changer les règles de l'autorité parentale.

\section{Conclusion}

Les propositions de lever l'anonymat des donneurs, de mettre fin à l'accouchement sous X, d'affaiblir l'adoption plénière et de la remplacer par l'adoption simple (open adoption), de personnifier les donneurs des gamètes (et par conséquent les gestations pour autrui), tout comme le soupçon de la monoparentalité, représentent ces habits nouveaux de l'ordre naturel.

La certitude de l'engendrement apparaît ainsi comme le rempart contre "l'individualisme et l'hédonisme narcissiste qui désintègre la société » (CNCE 2010). ${ }^{12}$ Comme le note le rapport Théry: vouloir fonder la filiation sur un acte volontaire est « le symptôme de la difficulté de l'idéologie individualiste contemporaine à reconnaitre que la famille est une institution, et à percevoir en conséquence la métamorphose de notre système symbolique de parenté " (Théry et Leroyer).

Et, lorsque cette famille nucléaire n'existe pas dans la réalité sociologique, il faut la réintroduire symboliquement à travers le statut des géniteurs. Cette entreprise constitue le degré le plus avancé dans le processus de biologisation du genre. En effet, l'hétérosexualité est ici réduite à sa dimension la plus élémentaire: le spermatozoïde et l'ovule. À partir de ce soubassement "glandulaire ", et en personnalisant le donneur jusqu'alors anonyme, la différence des sexes se trouverait finalement sauvegardée.

L'homoparenté est admise à condition qu'elle se soumette à ce nouvel ordre symbolique, c'est-à-dire que l'établissement de la filiation dans un couple homoparental n'institue pas un enfant comme "né " de ce couple, 
que la réalité biologique de l'engendrement ne soit pas occultée ainsi que le recommandent la CNCDH et le rapport Théry.

La famille homoparentale devient donc une forme de famille recomposée dont l'origine se trouve dans l'engendrement biologique.

Au-delà de la question familiale, cette entreprise de naturalisation de l'ordre des filiations n'a nullement comme objet la protection de l'intérêt de l'enfant in concreto mais le maintien in abstracto de l'ordre symbolique de la différence des sexes, autrement dit, la suprématie de l'hétérosexualité.

CERSA/CNRS Université de Paris II Panthéon Assas

\section{Notes}

1 Le 23 avril 2013, l'Assemblée nationale a adopté le projet de loi par un scrutin public. 566 députés ont participé au vote, 331 ont voté pour l'adoption du projet de loi, 225 ont voté contre, 10 se sont abstenus. A la suite de l'adoption du texte, soixante députés et soixante sénateurs de l'opposition ont saisi le Conseil constitutionnel qui a déclaré le texte conforme à la Constitution dans une décision du 17 mai 2013 (n² 2013-669). La loi n 2013-404 du 17 mai 2013, ouvrant le mariage aux couples de personnes de même sexe a été publiée au journal officiel le 18 mai 2013.

2 Le texte a été débattu à l'Assemblée nationale et au Sénat à partir du 29 janvier 2013. Les débats ont duré plus de 160 heures et 5,000 amendements furent discutés.

3 En effet, en décembre 2012, les députés socialistes prévoient de déposer un amendement autorisant la procréation médicalement assistée (PMA) pour les couples de femmes, le président de la République ayant laissé entendre qu'il ne s'y opposerait pas. Finalement, en janvier 2013, ils annoncent qu'ils ne déposeront pas d'amendement pour inclure la PMA dans le projet de loi sur le mariage homosexuel, acceptant qu'elle soit ajoutée à un autre projet de loi sur la famille, en mars 2013 reporté à octobre de la même année. Finalement le gouvernement a laissé le projet dans les mains de l'Assemblée Nationale (proposition de loi) qui devrait l'adopter en 2014 sans PMA pour les couples de même sexe.

4 Plus d'une centaine d'articles du code civil contiennent les termes père et mère malgré l'adoption de la loi sur le mariage et l'adoption pour les couples de même sexe.

$5 \mathrm{La}$ «Manif pour tous » est un collectif d'associations proches de l'Église catholique qui se présente comme apolitique malgré son ancrage à droite (voire à l'extrême droite). La "Manif pout tous » serait financée par Claude Bébéar (fondateur des assurances Axa), l'association anti avortement Vita, ou des associations catholiques de familles. Loin de l'image d'un mouvement spontané qu'elle voulait se donner, derrière la "Manif pour tous " se trouve l'américain Brian Brown, président de la très conservatrice National Organization for Marriage proche de l'Opus Dei.

6 L'adoption était considérée par l'Église comme une institution dangereuse car elle subvertissait l'engendrement naturel (Perreau 19).

7 Le juriste romain Iulius Paulus dans son Libro singulari de gradibus et adfinibus et nominibus eorum commence son traité sur la filiation en soulignant que "Tout jurisconsulte doit connaitre les degrés où se situent les parents et les alliés. D'abord, parce que les successions 
et les tutelles sont habituellement déférées par les lois à l'agnat le plus proche. Ensuite parce que le préteur, dans son édit, concède au parent le plus proche la possession des biens du défunt. En outre, la loi sur les juridictions criminelles publiques interdit de contraindre à témoigner malgré soi contre ses alliés et ses parents » (Legendre 1988 37).

8 Il n'existe pas de filiation biologique car toute filiation même celle provenant du fait biologique doit être prévue par la loi.

9 Le pédopsychiatre et psychanalyste Pierre Levy-Soussan, vice-président du Conseil national pour l'accès aux origines personnelles, le psychanalyste Jean-Pierre Winter et le psychiatre Christian Flavigny se sont vivement opposés au mariage pour tous au nom de la bonne structuration psychique de l'humain.

10 Toutefois, l'absence d'accès à l'AMP pour les couples de lesbiennes dans la loi Taubira a rassuré le Conseil d'Etat au point que dans une décision du 13 juin 2013 (n 362981), il déclare que "la règle de l'anonymat des donneurs de gamètes, qui est inscrite dans le code de la santé publique, le code civil et le code pénal et qui figure au nombre des principes fondamentaux de la bioéthique proclamés par la loi du 29 juillet 1994 et confirmés par la loi du 7 juillet 2011, n'est pas incompatible avec les stipulations de la convention européenne de sauvegarde des droits de l'homme et des libertés fondamentales (CEDH), et notamment son article 8 qui garantit le droit au respect de la vie privée et familiale ".

11 Agnès Fine et Geneviève Delaisi de Parseval considèrent dans ses travaux qu'il faut traiter l'homoparentalité comme une forme de pluriparentalité ou de coparentalité par le biais de la levée de l'anonymat des donneurs et la prééminence de l'adoption simple.

12 Le Comité consultatif national d'éthique s'est prononcé en 2010 contre la gestation pour autrui pour les couples d'hommes et contre le don de sperme pour les couples de femmes et les Sages de ce comité ont rappelé que la PMA était destinée à résoudre un problème de stérilité d'origine médicale et non à venir en aide à un choix de vie sexuelle, précisant que «l'ouverture d'une telle aide à l'homoparentalité (...) constituerait peut-être alors un excès de l'intérêt individuel sur l'intérêt collectif » et que "la médecine serait convoquée pour satisfaire un droit à l'enfant " (CNCE 31).

\section{Bibliographie}

Agacinski-Jospin, Sylviane. "L'homoparentalité en question, par Sylviane Agacinksi ». Le Monde. Web. <http://www.lemonde.fr/idees/article/2007/06/21/1-homoparentaliteen-question-par-sylviane-agacinski_926550_3232.html>. 21 Sept. 2014.

d'Avout, Louis. "La parenté homosexuelle à travers l'adoption: réflexions d'actualité ». Recueil Dalloz 31 (2012): 1973. Print.

Bertinotti, Domique. Présentation de la méthode d'élaboration du projet de loi sur la famille. Installation des quatre groupes de réflexion par Dominique Bertinotti, Ministre déléguée à la famille, 2013. Web. <http://www.social-sante.gouv.fr/IMG/pdf/DP_loi_famille_ 211013.pdf>. 21 Sept. 2014.

BFM TV. "Critiquée sur la Manif pour tous, BFM-TV vous donne rendez-vous le 27 janvier ». Le Nouvel Observateur et Rue 89. Web. <http://www.rue89.com/2013/01/14/ critiquee-sur-la-manif-pour-tous-bfm-tv-vous-donne-rendez-vous-le-27-janvier238579>. 21 Sept. 2014.

Borrillo, Daniel. "Le droit en matière de reproduction: une approche critique ". Reproduire le genre. Ed. Elsa Dorlin et Eric Fassin. Paris: Bibliothèque Publique d'information Centre Pompidou, 2010. 25-34. Print. 
—. «La parenté et la parentalité dans le droit: conflits entre le modèle civiliste et l'idéologie naturaliste de la filiation ". Reproduire le genre. Ed. Elsa Dorlin et Eric Fassin. Paris: Bibliothèque Publique d'information Centre Pompidou, 2010. 121-36. Print.

- " "La vérité biologique contre l'homoparentalité: le statut du beau-parent ou le "PaCS de la filiation" ». Droit et Société 72 (2009): 361-79. Print.

Code de la santé publique. Légifrance, France, 2014. Web. <http://legifrance.gouv.fr/ affichCode.do?cidTexte=LEGITEXT000006072665>. 21 Sept. 2014.

Commission Nationale Consultative des Droits de l'Homme (CNCDH). «Avis sur le projet de loi ouvrant le mariage aux couples de personnes de même sexe " (Assemblée plénière du 24 janvier 2013), Paris 2013. Web. <http://www.legifrance.gouv.fr/affichTexte. do?cidTexte=JORFTEXT000027415552>. 21 Sept. 2014.

Comité national consultatif d'éthique (CNCE) 2010. "Avis n 110 sur les problèmes éthiques soulevés par la gestation pour autrui (GPA)». Web. <http://www.ccne-ethique.fr/sites/ default/files/publications/avis_110.pdf>. 21 Sept. 2014.

Conseil d'Etat. Avis n 362981 du 13 juin 2013. Web http://legifrance.gouv.fr/affichTexte. do?cidTexte=JORFTEXT000027570259. 21 Sept 2014.

- Section du rapport et des études, Sciences de la Vie. De l'éthique au droit. Paris : La Documentation française, 1988. Print.

Cornu, Gérard. Droit civil. La famille. Paris: Montchrestien, 1993. Print.

Cour de Cassation, 2004. Web. <http://www.legifrance.gouv.fr/affichJuriJudi. do? oldAction $=$ rechJuriJudi\&idTexte $=$ JURITEXT000007469493\& fastReqId $=19049323$ 25\&fastPos=1>. 21 Sept. 2014.

Cour de Cassation (2012) Arrêt no 755 du 7 juin 2012 (11-30.261) - Cour de cassation Première chambre civile. Web. <http://www.courdecassation.fr/jurisprudence_2/ premiere_chambre_civile_568/755_7_23516.html>. 21 Sept. 2014.

Delaisi de Parseval, Geneviève. Famille à tout prix. Paris: Seuil, 2008. Print.

Ecoiffier, Matthieu. "Frigide Barjot Mobilise Les Antis Pour Le 26 Mai ». Libération. Web. $<$ http://www.liberation.fr/societe/2013/03/31/frigide-barjot-mobilise-les-antis-pourle-26-mai_892738>. 21 Sept. 2014.

"Etablissement de la filiation par possession d'état ». Service-Public.fr. Web. <http:// vosdroits.service-public.fr/F15395.xhtml>. 21 Sept. 2014.

Fassin, Éric. "Les "forêts tropicales" du mariage hétérosexuel. Loi naturelle et lois de la nature dans la théologie actuelle du Vatican ». Revue d'éthique et de théologie morale 261 (2010): 201-22. Print.

Fine, Agnès, et Agnès Martial. «Vers une naturalisation de la filiation ? "Genèses 78.1 (2010): 121-34. Print.

"La justice enjoint à une mairie de célébrer les mariages homosexuels ». Libération. Web. $<$ http://www.liberation.fr/societe/2014/07/10/la-justice-enjoint-a-une-mairie-decelebrer-les-mariages-homosexuels_1061365?xtor=rss-450>. 21 Sept. 2014.

Legendre, Pierre. Le dossier occidental de la parenté: textes juridiques indésirables sur la généalogie. Paris : Fayard, 1988. Print.

—. «Leçon IV ». L'inestimable objet de la transmission. Paris : Fayard, 1985. Print.

"Manif pour tous ». Web. <https://www.google.fr/search?q=pancartes+manif + pour+ tous\&tbm $=$ isch\&tbo $=u \&$ source $=$ univ\&sa $=$ X\&ei=_qYFVMnEG9PiaKqLgcAO\&ved $=0$ CC0QsAQ\&biw=1920\&bih=955>. 21 Sept. 2014.

Paul VI. Humanae Vitae. 25 juillet 1968. Print.

Petit, Cécile. Arrêt de la Cour de cassation 1ère Chambre civile, 28 mars 2000 (Bull. n 103), commentaire de l'avocat général.

Perreau, Bruno. Penser l'adoption. Paris: PUF, 2012. Print. 
Théry, Irène. «Anonymat des dons d'engendrement: le grand malentendu du débat français: Le Regard des Sciences Humaines et Juridiques ». SALF et Springer-Verlag France 2010. Web. <http://link.springer.com/article/10.1007/s12610-010-0064-5\#page-1> 21 Sept. 2014.

Théry, Irène, et Anne-Marie Leroyer. "Filiation, origines, parentalité. Le droit face aux nouvelles valeurs de responsabilité générationnelle ». Rapport au Ministère des affaires sociales et de la santé et au Ministère délégué chargé de la famille. Paris, La Documentation Française, 2014. Web. <http://www.ladocumentationfrancaise.fr/rapports-publics/ 144000203/>. 21 Sept. 2014. 